\title{
Abordagens Conceituais Contemporâneas na Geomorfologia Fluvial
}

\section{Contemporary Conceptual Approaches in Fluvial Geomorphology}

\author{
Mônica dos Santos Marçali \\ Universidade Federal do Rio de Janeiro \\ Rio de Janeiro, Brasil \\ Raphael Nunes de Souza Limaii \\ Universidade Federal do Rio de Janeiro \\ Rio de Janeiro, Brasil
}

\begin{abstract}
Resumo: A geomorfologia fluvial contemporânea se depara com questões desafiadoras, sobretudo, porque passa pela compreensão do período Holoceno Tardio/Antropoceno, que tem reflexos hoje e são intrinsecamente importantes para apreensão do impacto humano nos rios. Em razão das escalas em que operam as variáveis físicas em sistemas fluviais complexos, dois caminhos conceituais desenvolveram-se para analisar sua organização espacial e temporal. A visão de rede enfatizando controles na escala do sistema e a visão de trechos focando na descontinuidade e nos controles locais. A geomorfologia fluvial tem buscado compreender a organização dos sistemas fluviais complexos a partir da leitura integrada do paradigma da continuidade e descontinuidade. A visão integrada tem estimulado, no âmbito da geomorfologia, o surgimento de novos instrumentos teórico-metodológicos. Destaca-se a importância da integração das visões de rede e trechos na análise da organização de sistemas fluviais. O manejo dos rios e a gestão de bacias são processos contínuos que se referem tanto a um movimento sociocultural e modo de vida como um exercício técnico e científico.
\end{abstract}

Palavras-chave: Sistemas Complexos; Geomorfologia Fluvial; Organização Espacial; Escala Temporal; Gestão de Rios.

Abstract: Contemporary fluvial geomorphology faces challenging questions, especially as it goes by understanding the Late Holocene/Anthropocene period, which has repercussions today and are intrinsically important to understand the human river disturbance. Given the scale that physical rates operate in complex river systems, two conceptual paths were developed to analyze the spatial and temporal organization. The network view emphasizes controls on catchment-scale and a reach approach focuses on discontinuity and local controls. Fluvial geomorphology has seek to understand the organization

\footnotetext{
i Professora Associada do Departamento de Geografia. monicamarcal@ufrj.br.

ii Doutorando do Programa de Pós-Graduação em Geografia (PPGG/UFRJ). raphaelgeo85@gmail.com.
} 
of complex river systems from the integrated view of the continuity and discontinuity paradigm. This integrated approach has stimulated within the geomorphology, the emergence of new theoretical-methodological instruments. It is recognized that rivers management is an ongoing process, part of the socio-cultural development, which refers to both a social movement and scientific exercise.

Keywords: Complex Systems; Fluvial Geomorphology; Spatial Organization; Timescale; Management Rivers.

\section{Introdução}

A intenção desse artigo é propor uma discussão sobre o sistema fluvial, a partir de conceitos atuais referentes à sua organização espacial e temporal. Não se pretende fazer uma exaustiva revisão de metodologias e teorias, mas chamar a atenção para os paradigmas contemporâneos que orientam o estudo dos rios e, por conseguinte, as questões que envolvem as práticas de manejo, pondo em tela a disputa de concepções distintas sobre a forma como os rios deveriam ser e funcionar.

A geomorfologia fluvial, como uma subárea da ciência geomorfológica, tem seu possível surgimento no final do século XVIII, quando James Hutton e John Playfair reconheceram que os rios esculpiam seus próprios cânions, se houvesse tempo disponível (WHOL, 2014). Foi um período com importantes contribuições de naturalistas sobre relatos e descrições das paisagens, dando ideia de como se desenvolvia o curso dos rios. Outros marcos sucessivos, na maneira de pensar os rios, tiveram reflexos no surgimento e desenvolvimento da geomorfologia fluvial. Algumas dessas mudanças ficaram rotuladas de revolucionárias, correspondendo aos paradigmas davisiano, sistêmico e do aprofundamento das questões ambientais (VITTE, 2011). Atualmente, é também uma área essencial para a compreensão do processo de gestão e desenvolvimento de estratégias sustentáveis para o desenvolvimento, tendo se destacado por décadas como a maior subárea da geomorfologia no mundo (PIEGAY e KONDOLF, 2015; VAUDOR, 2015).

Nesta introdução optamos por destacar brevemente três momentos a fim de contextualizar as abordagens discutidas nos tópicos subsequentes. O primeiro, que abrange os séculos XVIII, XIX e início do século XX, caracteriza-se por ideias dominantes de catastrofismo versus uniformitarismo. Nessa linha destacam-se importantes trabalhos como o ciclo erosivo de Davis (1899) e o equilibrium e desenvolvimento da paisagem de Gilbert (1914). Esse contexto foi determinante para o real desenvolvimento da geomorfologia fluvial, mostrando que os rios se vinculavam com as ideias de equilibrium em um mundo desconectado do seu ambiente e analisados separadamente das influências de seu contexto ambiental. Isso iria persistir até o final da década de 1930.

Um segundo momento surge nos anos subsequentes, por volta de 1940-1950. A Segunda Guerra Mundial trouxe novidades tecnológicas na cartografia e na aerofotogrametria, que suscitaram novas questões sobre as formas e processos dos rios e serão fundamentais para o estabelecimento de novas bases paradigmáticas da geomorfologia fluvial. A visão evolucionista de Darwin, que havia predominado, passa então a incorporar uma visão sistêmica da paisagem, que se propõe compreender os mecanismos 
que regem os fluxos em uma bacia hidrográfica, a partir dos princípios da teoria geral dos sistemas (TGS). Os rios e as bacias hidrográficas passam a ser um conjunto único e indissociável, compondo um sistema fluvial.

Neste período, a geomorfologia fluvial aproximou-se ainda mais da hidrologia, da engenharia hidráulica e da geologia. Podemos simplificar que este foi um período onde se incorporou às leis de transporte dos rios a perspectiva da compreensão da bacia hidrográfica como um sistema aberto, consolidando a geomorfologia fluvial como um campo de pesquisa quantitativo. Nessa linha destacam-se importantes trabalhos como a ordem dos canais de Horton (1945) e Straler (1952), o índice de gradiente do rio de Hack (1957) e o nível de margens plenas de Wolman e Leopold (1957). De acordo com Smith (1993), a geomorfologia fluvial se estabelece como subdisciplina científica em 1964 com o livro clássico Fluvial Processes in Geomorphology, de Leopold Wolman e Miller.

O desenvolvimento da abordagem sistêmica nas análises ambientais foi fundamental para compreender a complexidade nas relações entre a natureza e a sociedade e a necessidade da abordagem integrativa do entendimento de natureza versus sociedade. A terminologia de geossistema foi cunhada por Sotchava (1977) para descrever a paisagem física como sistema composto por elementos topográficos, biogeográficos e pedológicos, dinamizados pelos fluxos climáticos e insere a ação das atividades humanas, sobretudo, destacando os níveis de complexidade nas interações de fluxos de energia e matéria. Exemplo disso é o clássico trabalho de Richard John Chorley - Geomorphology and General Systems Theory (1962) - onde ressalta a estreita analogia entre os clássicos sistemas abertos com as bacias de drenagem e destaca a complexidade de suas interações. Além deste, destacam-se também os trabalhos de Chorley e Kennedy (1971) e Schumm (1977).

A publicação do relatório intitulado "Os limites do crescimento", em 1972, elaborado por uma equipe do Instituto de Tecnologia de Massachusetts (MIT), ampliou os debates sobre a crise hídrica e as mudanças climáticas globais. Essas problemáticas passaram a interferir na busca de novas soluções para questões ambientais, dando o direcionamento nas pesquisas, juntamente com o advento de novas tecnologias. Talvez este seja o limiar para o surgimento de um terceiro momento de grande expressividade nas análises da geomorfologia fluvial contemporânea.

As noções de magnitude e frequência que modelam a forma dos rios (WOLMAN e GERSON, 1978), os ajustes de energia e a forma dos rios no tempo e no espaço (SCHUMM, 1973), e os efeitos do uso da terra nos rios (BRUNSDEN e THORNES, 1979), elevaram a complexidade com a qual os sistemas fluviais passaram a ser concebidos e analisados, trazendo diferentes possibilidades de entendimento da paisagem de um rio (CORRÊA, 2005).

O período Antropoceno surge para descrever a época geológica em que nós vivemos no presente e sugere que a Terra já deixou sua época geológica recente, referente ao último estado interglacial chamado Holoceno. E isso parece exigir uma mudança nas formas em que estudamos e entendermos as transformações ambientais (WHITEHEAD, 2014).

Nesse sentido, a geomorfologia fluvial contemporânea se depara com questões desafiadoras, sobretudo, porque passa pela compreensão do período Holoceno Tardio/ Antropoceno, que tem reflexos hoje e são intrinsecamente importantes para apreensão 
do impacto humano nos rios. Demandam, portanto, reflexões que envolvem conhecimentos sobre comportamento e mudanças nas trajetórias dos rios, fundamentais para se buscar respostas para o manejo e gestão de bacias hidrográficas.

\section{Escalas, Tempo e Mudanças no Sistema Fluvial}

A ideia de organização e a ideia de sistema, são ainda, não apenas embrionárias, mas dissociadas. Eu me proponho aqui a associa-las, já que o sistema é o caráter fenomenal e global do qual se revestem as inter-relações cuja disposição constitui a organização do sistema. (MORIN, 2013, p. 135)

A organização de um sistema complexo corresponde à disposição das inter-relações de seus elementos que produz uma unidade complexa ou sistema. Seu estudo busca um meio de estimar a probabilidade de um conjunto de variáveis ou elementos em interação se auto-organizarem (MORIN, 2013). O autor problematiza o conceito de sistema como um conceito-piloto, que pode ser considerado uma unidade complexa organizada resultante das interações entre um observador e o universo fenomênico, que permite representar e conceber unidades complexas constituídas por inter-relações organizacionais entre elementos. A noção de sistemas não é simples e nem absoluta, comporta na sua unidade, relatividade, dualidade, multiplicidade, cisão, antagonismo e o problema da sua inteligibilidade abre a problemática da complexidade (MORIN, op. cit.). Portanto, a organização transforma, produz, liga e mantém o sistema.

Em sistemas fluviais, a complexidade de processos geomorfológicos, hidrológicos e bióticos operando sobre múltiplas escalas espaciais e temporais produzem notável diversidade de ambientes ribeirinhos (POOLE, 2002). Embora a heterogeneidade espacial garanta que a composição biofísica de todos os sistemas apresentem especificidades, muitas relações espaciais e padrões se repetem entre sistemas (CULLUM et al., 2008).

Em razão das escalas em que operam as variáveis físicas em sistemas fluviais, dois caminhos conceituais desenvolveram-se para analisar sua organização espacial e temporal. De um lado, uma visão de rede (enfatizando controles na escala do sistema), de outro, uma visão de trechos (focando na descontinuidade e nos controles locais) (PETTS e AMOROS, 1996; Schumm, 1977). Para Cullum et al. (2008), essas duas perspectivas compõem o paradigma da continuidade e descontinuidade. Segundo os autores, enquanto a primeira oferece modelos de interpretação preditivos da rede, a segunda permite aumentar a precisão das interpretações na escala do canal (Figura 1).

$\mathrm{Na}$ visão de rede destacam-se duas abordagens de análise que idealizam padrões e controles no nível do sistema. As relações fractais na geometria da rede e as dimensões do sistema fluvial.

A geometria fractal foi introduzida por Mandelbrot (1983) para descrever padrões significativamente complexos de distribuição, estrutura e dinamismo da natureza, ou seja, não lineares, irregulares, caóticos e aleatórios. O conceito de dimensão fractal surge como uma das ferramentas propostas para a análise dos sistemas complexos, sendo definida como a medida do grau de irregularidades em diferentes escalas; e é relacionada com o aumento da medida de um objeto, enquanto a escala do instrumento de medida 


\section{Paradigmas}

\section{Continuidade x Descontinuidade}
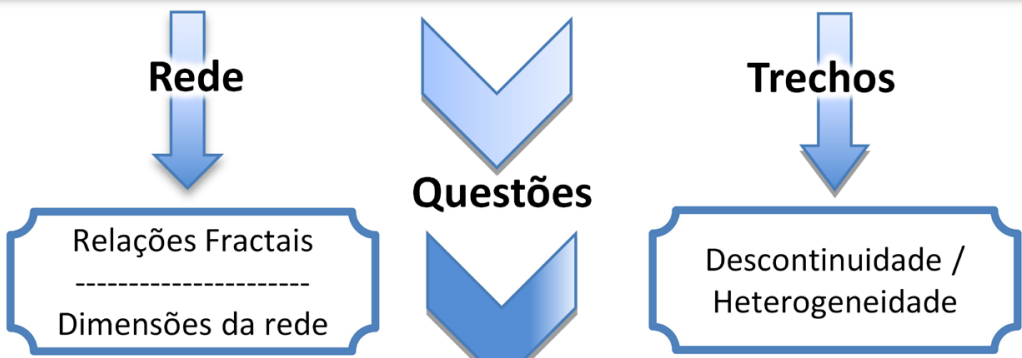

\section{Ambientais}

\section{Dinâmica Hierárquica de Trechos}

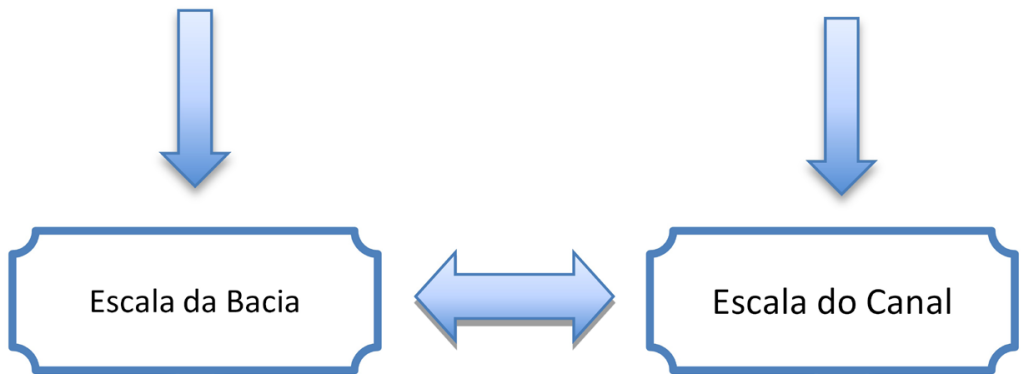

Figura 1 - Paradigmas contemporâneos da organização espacial de sistemas fluviais. Elaboração dos autores. 
diminui (MURRAY et al., 2014). Uma bacia de drenagem inclui em suas transformações bacias menores ao longo de seus afluentes. Na natureza, esse processo termina com as bacias de drenagem de primeira ordem, cujos tamanhos determinam a fractalidade da escala interna (VESTENA e KOBYAMA, 2010).

$\mathrm{Na}$ tentativa de melhor explicar a estrutura e função das comunidades biológicas e dos gradientes físicos nos sistemas fluviais, desenvolveram-se conceitos voltados para a visão ecológica do rio, passando a analisar interações de processos geomorfológicos e hidrológicos, a partir de quatro dimensões fluviais (WARD, 1989; WARD e STANFORD, 1995). A dimensão longitudinal compreende as relações montante-jusante e tributário-tronco (continuum fluvial; VANNOTE et al., 1985; MONTGOMERY, 1999); a dimensão lateral contém relações encosta-canal e canal-planície (pulso de inundação; JUNK et al., 1989); a dimensão vertical inclui níveis de inundação e interações superfície-subsuperfície (corredores hiporrêicos; STANFORD e WARD, 1993); e, por último, a dimensão temporal que compreende magnitude, frequência e sincronismo do movimento da água e sedimentos, regimes de perturbação e padrões decorrentes de perturbações (LEOPOLD et al., 1964; SCHUMM, 1977).

Ao passo que o conceito de continuidade fluvial (originalmente RCC: River Continuum Concept) propõe o entendimento de padrões consistentes de estrutura e função (física e biológica) ao longo do curso de um rio, o conceito de descontinuidade fluvial (originalmente SDC: Serial Discontinuity Concept) aponta para transições abruptas entre segmentos do rio, resultantes de efeitos de construção de barragens, variações locais ou regionais de litologia, geomorfologia, clima e vegetação (LANA e CASTRO, 2006).

Os exercícios de colocar cada dimensão em perspectiva, isolando relações e atributos do sistema, contribuem para a análise de movimentos e conexões específicas, replicáveis em diferentes escalas nos sistemas fluviais. No entanto, isoladamente, a visão de rede não é capaz de descrever completamente a variabilidade presente em um sistema fluvial, visto que, o comportamento dos canais é fortemente influenciado pela história de um local específico, comumente desconsiderada em modelos determinísticos (MURRAY et al., 2014).

Na visão de trechos, é dado enfoque às análises de descontinuidades e controles locais que produzem heterogeneidades na paisagem. As feições que ocorrem nesta escala são resultantes diretas das interações entre fluxo e sedimentos, refletindo a distribuição de energia ao longo de um trecho do rio. Essas relações podem apresentar elevada variabilidade temporal, de acordo com o regime hidrológico do rio. A interação hidráulica entre partículas de diferentes composições e calibres permite o estabelecimento de uma matriz de ambientes para sustentar uma variedade de organismos bentônicos. Da mesma maneira, o tamanho do substrato, sua heterogeneidade, a frequência e o volume de retrabalhamento dos sedimentos são determinantes, por exemplo, para a diversidade e abundância de populações de invertebrados (BRIERLEY e FRYIRS 2005; WU e LOUCKS, 1995).

Novas questões ambientais passaram a demandar a integração destas duas perspectivas (visões de trecho e rede), aliando as análises de atributos físicos observáveis com a previsão de comportamentos e trajetórias de mudança dos rios (NEWSON, 2002). Exemplos disso são as relações entre mudanças de uso do solo com assoreamento e inundação de canais, ocorridos desde períodos coloniais; intervenções de engenharia como retifica- 
ções, represamentos e irrigações ocorridos em processos recentes de expansão urbana e agrícola; e relações das mudanças climáticas com magnitude e frequência de processos.

Para lidar com essas questões, as pesquisas em geomorfologia fluvial vêm adotando o paradigma para análise da organização dos sistemas fluviais, denominado de dinâmica hierárquica de trechos (FRISSEL, 1986). Essa perspectiva deriva das ideias da teoria da hierarquia, originada na ecologia, na qual os sistemas são apreendidos como elementos dispostos em diferentes níveis de organização. Elementos em níveis mais elevados na hierarquia restringem atributos e comportamento em níveis mais baixos (ALLEN e STARR, 1982).

Para a interpretação de sistemas fluviais, destacam-se dois níveis escalares hierárquicos (POOLE, 2002). O primeiro representa a escala da bacia (regional) e tem como principal objetivo organizar informações sobre a estruturação da rede de drenagem, considerando os principais fatores de controle, sendo eles: o perfil longitudinal do canal, parâmetros morfométricos (padrão de drenagem, densidade, hierarquia e forma da bacia), análise do regime hidrológico e caracterização ambiental regional (geologia, geomorfologia, solos, topografia, clima e uso do solo) (NEWSON e NEWSON, 2000).

O segundo nível de análise corresponde à escala do canal (local), que permite acompanhar fenômenos que ocorrem em níveis de detalhe, fundamentais para a distinção de ambientes fluviais e estabelecimento dos limites onde eles operam. Nesta escala é possível levantar informações sobre a característica do vale; feições morfológicas no canal e sua forma em planta; e material do leito (BISSON e MONTGOMERY, 1996).

Diante deste novo paradigma, surgem ao longo da década de 1990 novos modelos de classificação e análise de rios visando identificar as relações entre processos e formas, sob a perspectiva de que estes ocupam um lugar e função dentro do contexto da paisagem e da bacia hidrográfica (NAIMAN, 1992; ROSGEN, 1994; BRIERLEY e FRYIRS, 2005). Nestes modelos ocorrem esforços metodológicos para integrar as escalas, refletindo o reconhecimento de que um rio faz parte de um sistema físico com uma história evolutiva. Essa marca do passado é comumente referida como herança, antecedência, memória do sistema, ou contingência histórica, compreendendo as pré-condições de respostas do sistema para eventos de perturbações contemporâneas (MIKA et al., 2010; SIMON e RINALDI, 2006; GOUDIE, 1993).

Diversos pesquisadores passaram, ainda, a estudar os sistemas fluviais através de seus ajustes e mudanças progressivas a partir de diferentes escalas de tempo, que vão desde pequenas variações sazonais às profundas transformações de escala geológica (BRIERLEY e FRYIRS, 2005).

Durante longos períodos, influências geológicas como tectônica e controle de nível de base conduzem à evolução da paisagem, moldando o relevo, a declividade, o confinamento do vale e padrões de erosão e sedimentação (Ab'SABER, 1950; BIGARELLA, 1965; SCHUMM e LITCHY, 1965). Estas correspondem às condições de limite impostas ao sistema. Em contraste, influências climáticas definem as condições de limite do fluxo dentro das quais os rios operam. Ou seja, interações entre fluxo, sedimento e vegetação determinam o regime comportamental dos trechos de rios (MURRAY et al., 2014; FRYIRS e BRIERLEY, 2010). Mudanças climáticas podem alte- 
rar as condições de limite, possibilitando a criação de um novo tipo de rio com uma capacidade de ajuste diferente (BRIERLEY e FRYIRS, 2005). Exemplo deste processo é o modelo teórico de Bigarella e Mousinho (1965) acerca das oscilações climáticas pleistocênicas no reafeiçoamento do relevo tropical do sudeste brasileiro e formação de depósitos correlativos regionais.

Em escalas de tempo mais curtas, os rios estão sujeitos a um conjunto de eventos impactantes, tais como inundações, secas e queimadas. Tais eventos são concebidos como perturbações ao sistema fluvial, sendo definidas como uma mudança na intensidade dos processos, referindo-se a qualquer fator que afete as condições de limite (OWENS et al., 2010; BOLATTI et al., 2014). Eventos de perturbação são, portanto, aqueles que interferem nas taxas de ajustes podendo incorrer em mudanças nos rios (GAEUMAN et al, 2005). Porém, as taxas nas quais os rios se ajustam variam de sistema para sistema e ao longo de diferentes períodos de tempo, de maneira que é necessária diferenciação entre os conceitos de ajuste, comportamento e mudança.

Em sistemas fluviais, a capacidade de ajuste é uma medida da variabilidade potencial e extensão de ajustes geomorfológicos que podem ocorrer ao longo de um rio (BRIERLEY e FRYIRS, 2005). O comportamento fluvial reflete ajustes geomorfológicos e ecológicos em curso ao longo de períodos nos quais as condições de contorno de fluxo permanecem relativamente uniformes (por exemplo, regime de descarga sólida e líquida), de maneira que um trecho mantenha uma configuração característica da relação de forma e processo. A mudança fluvial, por outro lado, ocorre quando um segmento do rio apresenta uma substituição indiscriminada em sua relação de forma e processo estabelecida (SCHUMM, 1969). Isto pode ser induzido por uma perturbação de ordem natural ou antrópica.

Ocasionalmente, os rios são sujeitos a mudanças dramáticas e quase instantâneas em resposta a eventos catastróficos. Em alternativa, se o sistema se encontra perto de uma condição de limite, eventos relativamente pequenos podem reconfigurar o sistema, transformando-o (SCHAEFFER et al., 2001). Desse modo, as mudanças em um rio podem ocorrer quando fatores intrínsecos (p. ex., declividade) e extrínsecos (p. ex., clima) transgridam abruptamente a condição de limite, bem como através de ajustes progressivos ( $p$. ex., desmatamento).

Lake (2000) descreve a variabilidade do ritmo e magnitude dessas perturbações, que se distinguem amplamente de sistema para sistema. Pulso de Perturbação são os eventos episódicos de baixa frequência, alta magnitude e duração limitada (p. ex., inundações sazonais), cujos efeitos tendem a ser localizados. Impactos fora do local de ocorrência são mínimos e breves, assim como a reorganização do material do leito dentro do trecho do rio após a enchente. Perturbação de Pressão corresponde à alteração persistente e contínua das condições de limite do fluxo (p. ex., construção de uma barragem). Tais eventos e suas respostas ocorrem sobre áreas maiores do que o tipo Pulso de Perturbação, e as respostas não são espacialmente uniformes e tendem a ser mais permanentes. Por último, Perturbação Progressiva são eventos cuja força de sua influência cresce continuamente ao longo do tempo e do espaço. Exemplos incluem secas prolongadas, que promovem a sedimentação no leito, e perda progressiva de vegetação nativa, que induzem mudanças na hidrologia de encostas. 
Para Brunsden e Thornes (1979), além dos tipos de impactos, é necessário compreender as respostas do sistema a eventos de perturbação individuais, pois são seus efeitos cumulativos que determinam a trajetória evolutiva do rio.

Os tipos de respostas do sistema fluvial aos diferentes tipos de perturbação variam, refletindo as condições da paisagem e a conectividade do sistema (HOOKE, 2003). O conceito de conectividade é definido como a transferência de matéria e energia entre diferentes compartimentos do sistema (BRIERLEY et al., 2006). Em paisagens conectadas, os efeitos de perturbação são frequentemente propagados através delas. Em contraste, respostas às perturbações são ineficientemente propagadas através de paisagens desconectadas. Barragens, por exemplo, amortecem fluxos de água e sedimentos, absorvendo os impactos (FRYIRS et al., 2007).

Brunsden e Thornes (1979) propõem três categorias para analisar os tipos de respostas em sistemas, sendo elas o tempo de reação, tempo de relaxamento e tempo de recuperação. O tempo de reação é definido como o tempo necessário para que o sistema responda à alteração de intensidade de um processo. Tempo de recuperação compreende uma medida da capacidade geral do sistema para retornar ao seu estado antecedente, ou alcançar uma forma característica entre eventos de perturbação. Dependendo da sensibilidade do sistema às perturbações e o tempo entre estes eventos, várias formas de recuperação podem ocorrer. A rápida recuperação ocorrerá em sistemas autoajustáveis e, nesse caso, o sistema é capaz de se adaptar muito facilmente e rapidamente após a perturbação. A reação e o tempo de relaxamento são rápidos. A demora na recuperação de um distúrbio ocorre após um intervalo de tempo. O tempo de reação pode ser rápido, mas o sistema leva algum tempo para voltar ao seu estado anterior. Alguns distúrbios podem forçar um sistema além de sua capacidade de manter ou recuperar a sua forma. Nestes casos, o sistema se ajusta para uma condição de fluxo alterada e uma nova configuração é formada, onde eventos podem deixar uma impressão persistente sobre a paisagem.

Cheias de diferentes magnitudes, frequência e duração proporcionam diferentes formas e taxas de ajustes geomorfológicos para diferentes tipos de rios. A análise da relação magnitude-frequência de cheias e a avaliação do histórico de vazões são necessárias para interpretar quais eventos moldam e ajustam dentro de uma morfologia característica e os eventos que alteram a trajetória evolutiva de um segmento do rio. O conhecimento dessas relações é de grande relevância para elaboração de prognósticos de ajustes fluviais e mudanças.

\section{Novos Paradigmas para Trabalhar na Gestão dos Rios}

Pode-se considerar o estado natural como uma forma de estado histórico do rio, uma condição de referência ou uma preconcepção artificial de como o rio deveria se parecer? (Tradução de Fryirs e Brierley, 2009, p. 2).

O planejamento e o manejo de rios ao redor do mundo têm trazido para a agenda da gestão pública a discussão de duas vertentes teóricas e conceituais, que põem em disputa o 'design' dos rios, isto é, a maneira como os rios devem ser e funcionar (BRIERLEY e FRYIRS, 2008). De um lado, um modelo tradicional, iniciado por volta do século XVIII, 
preconizando a domesticação dos rios através da adaptação do fluxo e modificação do seu comportamento para condições desejadas; de outro, um modelo mais recente, surgido em meados do século XX, que busca alcançar um estado natural dos sistemas fluviais adaptando e orientando as ocupações à dinâmica natural dos rios. Em outras palavras, "o rio do meu jeito" versus "o rio do jeito do rio" (WILLIAMS, 2001).

O modelo de domesticação abrange estratégias de drenagem das vazões pelo caminho mais curto e com a maior velocidade de escoamento possível. Tal premissa tem como objetivos principais ganhar novas terras para a agricultura e urbanização, bem como minimizar os efeitos locais das cheias. Por décadas, as obras com base nesta concepção, sistematicamente, apresentaram consequências não consideradas ou avaliadas no planejamento, como: ajustes e mudanças nos processos geomorfológicos, redução da variedade de biota e prejuízos materiais e humanos cada vez maiores em função de cheias (JAMES e MARCUS, 2006).

Já o modelo de condição natural trabalha com objetivos que priorizam o reestabelecimento mais próximo possível da biota e preservação das áreas naturais de inundação, através do manejo regular ou de programas de gestão orientando práticas que viabilizem tais funções (ROSGEN, 2006).

Neste contexto, destaca-se a importância da integração das visões de rede e trechos na análise da organização de sistemas fluviais, sendo estas, maneiras de preparar o olhar de pesquisadores e envolvidos no processo de gestão para analisar criticamente arcabouços de "soluções prontas" (MIKA et al., 2010). Isso contribui para se evitar equívocos nas tomadas de decisão decorrentes da generalização de conhecimentos e transferência de descobertas para fora do seu contexto original.

Para Brierley e Fryirs (2009) e Brierley (2010), o manejo de rios deve buscar reconectar a sociedade ao lugar (cotidiano emocionalmente envolvido contido na dinâmica geral de espaço-tempo de processos socioecológicos) e promover prospectos para melhorar a "saúde" do rio (integridade de ecossistemas aquáticos e sistemas geomorfológicos), tornando-se parte do desenvolvimento sociocultural. Segundo esses autores, o manejo de rios é, portanto, ao mesmo tempo, um processo social e técnico.

Nesta perspectiva, as questões que permeiam as tomadas de decisão precisam ser respondidas para cada caso, tendo-se em conta as características de cada bacia e de cada canal, refletindo a trajetória de ajuste dos rios e dos valores da própria sociedade perante os seus recursos naturais (HOOKE, 2003; BROOKES, 1987).

No Brasil, apesar da existência de leis que dispõem sobre zoneamento e o ordenamento do uso e ocupação do território, inundações frequentes e problemas sociais graves relacionados à habitação e saneamento afetam, ainda nos dias de hoje, populações em diversos municípios. A exemplo dos rios da região norte do estado do Rio de Janeiro, onde o processo de uso e ocupação incorreu em diversos impactos diretos (modificações diretas no canal, como retificações e represamentos) e indiretos (atividades humanas realizadas fora da área dos canais, mas que modificam o comportamento do rio), resultantes de décadas de um modelo predominantemente de domesticação na gestão ambiental (MARÇAL, 2013; LIMA e MARÇAL, 2013; ASSUMPÇÃO e MARÇAL, 2012).

Nesse sentido, devemos observar que o período Antropoceno envolve o conjunto das mudanças provocadas nos sistemas fluviais, que produziram, dentre outros efeitos, 
o aprofundamento da crise hídrica, que hoje, desafiam a geomorfologia fluvial a compreender, a partir dos arcabouços conceituais contemporâneos, as trajetórias de evolução dos rios.

\section{Conclusão}

O estudo de sistemas complexos tem evoluído nos últimos 30 anos, desenvolvendo e demandando novos conceitos, ferramentas e maneiras de compreender antigos fenômenos. As recentes mudanças em nossas perspectivas de observações sobre causa e efeito estão ajudando a expandir nosso olhar e reflexões para novas explicações possíveis de eventos da paisagem, suas estruturas e comportamentos.

$\mathrm{Na}$ literatura científica, tem-se observado um número crescente de pesquisas voltadas ao entendimento da diversidade e o dinamismo dos sistemas complexos e suas respostas frente às perturbações causadas pelo homem. Nessa perspectiva, a geomorfologia fluvial contemporânea tem buscado compreender a organização dos sistemas fluviais, a partir da leitura integrada do paradigma da continuidade e descontinuidade. Esta necessidade de uma visão integrada tem estimulado, no âmbito da geomorfologia, o surgimento de novos instrumentos teórico e metodológicos.

Nesse sentido, a compreensão dos sistemas fluviais a partir dinâmica hierárquica dos trechos será importante para colocar em perspectiva as diferentes escalas espaciais e temporais em que operam o sistema fluvial. Basicamente, os processos e formas fluviais vêm sendo cada vez mais identificados e interpretados a partir da estruturação e organização das escalas espaciais e temporais em uma rede hierarquizada. Desse modo, os novos modelos explicativos desenvolvem-se a partir das relações entre as condições de limite do sistema e os componentes de trechos de canal-planície para determinar as formas e comportamentos resultantes.

Considerando que a geomorfologia fluvial tem interseção com outras áreas (geologia, engenharia hidráulica e ecologia), faz com que os conceitos trabalhados nesta disciplina, também alcancem e sejam alcançados por outras áreas cientificas que contribuem para a prática do manejo e gestão dos rios, na perspectiva dos sistemas complexos.

Abordagens relativas à preservação e manejo dos rios variam significativamente de lugar para lugar e as tentativas de melhorar a saúde do rio estão subordinadas aos esforços de gerir o espaço e o uso da água de forma mais eficaz, minimizando as consequências antrópicas negativas nos sistemas fluviais. O manejo de rios e a gestão de bacia são, portanto, um processo contínuo, que se refere tanto a um movimento social e modo de vida como um exercício técnico e científico.

\section{Referências Bibliográficas}

AB'SABER. A Serra do Mar e a Mata Atlântica em São Paulo. Bol. Paulista Geografia, v. 2, n. 4, p. 61-70, 1950.

ALLEN, T.; STARR, T. Hierarchy. Chicago, IL: University of Chicago Press, 1982. 
BISSON, P. A.; MONTGOMERY, D. R. Valley Segments, Stream Reaches and Channel Units. In: HAUER, R. F. R.; LAMBERT, G. A. (Eds.). Methods in Stream Ecology. Londres: Academic Press, 1996, p. 23-52.

ASSUMPÇÃO, A. P.; MARÇAL, M. S. Retificação dos canais fluviais e mudanças geomorfológicas na planície do Rio Macaé (RJ). Revista de Geografia, Recife, v. 29, p. 19-36, 2012.

BIGARELLA, J. J; ANDRADE, G. O. Contribution to the Study of the Brazilian Quaternary. In: WRIGHT, H. E. Jr.; FREY, D. G. (Eds.). International Studies on the Quaternary. Geol. Soc. Am. Spec. Papers. New York, 84, 1965, p. 433-451.

; MOUSINHO, M. R. M. Considerações a respeito dos terraços fluviais, rampas de colúvio e várzeas. Boletim Paranaense Geografia, n. 16/17, p. 153-197, 1965.

BOLLATI, I.M.; PELLEGRINI, L.; RINALDI, M.; DUCI, G.; PELFINI, M. Reach-Scale Morphological Adjustments and Stages of Channel Evolution: the Case of the Trebbia River (northern Italy). Geomorphology, v. 221, p. 176-186, 2014.

BRIERLEY, G. J.; FRYIRS, K. A. Geomorphology and River Management: Applications of the River Styles Framework. Oxford, UK: Blackwell Publishing, 2005.

; FRYIRS, K. Don't Fight the Site: Three Geomorphic Considerations in Catchment-Scale River Rehabilitation Planning. Environmental Management, v. 43, n. 6, p. 1201-1218, 2009.

; S Space, Place and a Healthy Dose of Realism Grounding the Process of River Repair. 4h ECRR Conference on River Restoration. Italy, Venicltália, Veneza, Ilha de S. Servolo, p. 16-21, 2008.

; _ _ JAIN, V. Landscape Connectivity: The Geographic Basis of Geomorphic Applications. Area, v. 38, n. 2, p. 165-174, 2006.

Landscape Memory: The imprint of the Past on Contemporary Landscape Forms and Processes. Area, v. 42, n. 1, p. 76-85, 2010.

BROOKES, A. Restoring the Sinuosity of Artificially Straightened Stream Channels. Environmental Geology and Water Science, v. 10, p. 33-41, 1987.

BRUNSDEN, D.; THORNES, J.B. Landscape Sensitivity and Change. Translates of the Institute of British Geographers, v. NS4, p. 463-484, 1979.

CHORLEY, R. Geomorphology and general systems theory. U.S. Geological Survey Professional Paper, n. 500B, p. 1-10, 1962. 
; KENNEDY, B. A. Physical Geography: a Systems Approach. Londres: Prentice-Hall, 1971.

CORRÊA, A. C. B. A Geografia Física: uma pequena revisão de seus enfoques. Rios, v. 1, p. 170-181, 2005.

CULLUM, C.; BRIERLEY, G. J; THOMS, M. The Spatial Organization of River Systems. In: BRIERLEY, G. J.; KIRSTIE, A. (Eds.) River Futures: an Integrative Scientific Approach To River Repair. Island Press, Society for Ecological Restoration International, p. 43-64, 2008.

DAVIS, W. M. The Geographical Cycle. Geographical Journal of Royal Society, n. 14, p. 481-504, 1899.

FRISSELL, C. A.; LISS, W. J.; WARREN, C. E.; HURLEY, M. D. A Hierarchical Framework for Stream Habitat Classification: Viewing Streams in a Watershed Context. Environmental Management, v. 10, n. 2, p. 199-214, 1986.

FRYIRS, K.; BRIERLEY, G. J. Naturalness and Place in River Rehabilitation. Ecology and Society, v. 14, n. 1, p. 20, 2009.

; ___ Antecedent Controls on River Character and Behaviour in Partly Confined Valley Settings: Upper Hunter Catchment, NSW, Australia. Geomorphology, v. 117, n. 1-2, p. 106-120, 2010.

; PRESTON, N. J.; KASAI, M. Buffers Barriers and Blankets: the (Dis)Connectivity of Catchment-Scale Sediment Cascades. Catena, v. 70, p. 49-67, 2007.

GAEUMAN, D.; SCHMIDT, J. C.; WILCOCK, P. R. Complex Channel Responses to Changes in Stream Flow and Sediment Supply on the Lower Duchesne River, Utah. Geomorphology, v. 64, n. 3-4, p. 185-206, 2005.

GILBERT, G. K. The Transportation of Debris by Running Water. US Geological Survey Professional Paper, n. 86, 1914.

GOUDIE, A. The Human Impact: Man's Role in Environmental Change. Oxford: Blackwell, 1993.

HACK, J. T. Studies of longitudinal profiles in Virginia and Maryland. U.S. Geological Survey Professional Paper 294-B, Washington DC, 1957.

HOOKE, J. Coarse Sediment Connectivity in River Channel Systems: a Conceptual Framework And Methodology. Geomorphology, v. 56, n. 1-2, p. 79-94, 2003. 
Mônica dos Santos Marçal e Raphael Nunes de Souza Lima

HORTON, R. E. Erosional Development of Streams and their Drainage Basins: Hydrophysical Approach to Quantitative Morphology. Geological Society of America Bulletin, v. 56, n. 3, p. 275-370, 1945.

JAMES, L. A.; MARCUS, W. A. The Human Role in Changing Fluvial Systems: Retrospect, Inventory and Prospect. Geomorphology, v. 79, n. 3-4, p. 152-171, 2006.

JUNK, W. J.; BAYLEY, P. B.; SPARKS, R. E. The Flood Pulse Concept in River-Floodplain Systems. In: DODGE, D. P. (Ed.). Proceedings of the International Large River Symposium. Can. Spec. Public. Fish. Aquat. Sci., n. 106, p. 110-127, 1989.

LAKE, P. S. Disturbance, patchiness, and diversity in streams. Journal of the North American Benthological Society, v. 19, p. 573-46, 2000.

LANA, C.; CASTRO, P.T. Respostas da rede de drenagem à heterogeneidade geológica em bacias hidrográficas: uma comparação entre as bacias do Alto Rio das Velhas e Jequitaí - MG. In: SIMPÓSIO NACIONAL DE GEOMORFOLOGIA, 6, Goiânia. Anais. Goiânia, Brasil, 2006.

LEOPOLD, L. B.; WOLMAN, M. G.; MILLER, J. P. Fluvial Process in Geomorphology. San Francisco: W. F. Freeman and Co., 1964.

LIMA, R. N. S.; MARÇAL, M. S. Avaliação da Condição Geomorfológica da Bacia do rio Macaé - RJ a partir da Metodologia de Classificação dos Estilos Fluviais. Revista Brasileira de Geomorfologia, v. 14, p. 171-179, 2013.

MANDELBROT, B. B. The fractal geometry of nature. San Francisco: W. H. Freeman and Co., 1983.

MARÇAL, M. S. Análise das mudanças morfológicas em seções transversais ao Rio Macaé - RJ. Revista Brasileira de Geomorfologia, v. 14, p. 59-68, 2013.

MIKA, S.; HOYLE, J.; KYLE, G.; HOWELL, T.; WOLFENDEN, B.; RYDER, D.; KEATING, D.; BOULTON, A.; BRIERLEY, G.; BROOKS, A. P.; FRYIRS, K.; LIESHMAN, M.; SANDER, M.; ARHINGTON, A.; CREESE, R.; DAHM, M.; MILLER, C.; PUSEY, B.; SPINK, A. Inside the "Black Box" of River Restoration: Using Catchment History to Identify Disturbance and Response Mechanisms to Set Targets for Process-Based Restoration. Ecology and Society, v. 15, n. 4, p. 8, 2010.

MONTGOMERY, D. R. Process Domains and the River Continuum. Journal of the American Water Resources Association, v. 35, n. 2, p. 397-410, 1999.

MORIN, E. O Método 1. A natureza da natureza. 3. ed. Porto Alegre: Sulina, 2013. 
MURRAY, A. B.; COCO, G.; GOLDSTEIN, E. B. Cause and Effect in Geomorphic Systems: Complex Systems Perspectives. Geomorphology, v. 214, p. 1-9, 2014.

NAIMAN, R. J.; LONZARICH, D. G; BEECHIE, T. J. General Principles of Classification and the Assessment of Conservation Potential of Rivers. In: BOON, P. J.; CALOW, P.; PETTS, G. E. (Eds.). River Conservation and Management. Chichester: Wiley, 1992, p. 93-123.

NEWSON, M. D. Geomorphological Concepts and Tools for Sustainable River Ecosystem Management. Aquatic Conservation: Marine and Freshwater Ecosystems, v. 12, n. 4, p. 365-379, 2002.

NEWSON, M. D.; NEWSON, C. L. Geomorphology, Ecology and River Channel Habitat: Mesoscale Approaches to Basin-Scale Challenges. Progress in Physical Geography, v. 24, p. 195-221, 2000.

OWENS, P. N.; PETTICREW, E. L.; VAN DER PERK, M. Sediment Response to Catchment Disturbances. Journal of Soils and Sediments, v. 10, n. 4, p. 591-596, 2010.

PETTS, G. E.; AMOROS, C. Fluvial Hydrosystems. Londres: Chapman \& Hall, 1996.

PIÉGAY, H.; KONDOLF, M.; MINEAR, T.; VAUDOR, L. Trends in Publications in Fluvial Geomorphology Over Two Decades: A Truly New Era in the Discipline Owing to Recent Technological Revolution? Geomorphology, v. 248, p. 489-500, 2015.

POOLE G. C. Fluvial Landscape Ecology: Addressing Uniqueness Within the River Discontinuous. Freshwatter Biology, v. 47, p. 641-660, 2002.

ROSGEN, D. L. River Restoration Using a Geomorphic Approach FOR Natural Channel Design. PROCEEDINGS of the Eighth Federal Interagency Sedimentation Conference (8thFISC), p. 394-401, 2006.

ROSGEN, D. L. The classification of natural rivers. Catena, v. 22, p.169-199, 1994.

SCHEFFER, M. S.; CARPENTER, J. A.; FOLEY, C. Catastrophic shifts in ecosystems. Nature, n. 413, p. 591-96, 2001.

SCHUMM, S. A., River Metamorphosis. Proceedings of the American Society of Civil Engineers. Journal of the Hydraulics Division, v. 95, p. 255-273, 1969.

The Fluvial System. Chichester: J. Wiley \& Sons, 1977.

. Geomorphic Thresholds and Complex Response of Drainage Systems. In: MORISAWA, M. (Ed.). Fluvial Geomorphology. Nova York: SUNY Binghamton Publications in Geomorphology, 1973. 
; LICHTY, R W. Time, Space and Causality in Geomorphology. American Journal of Science, v. 263, p. 110-119, 1965.

SIMON, A.; RINALDI, M. Disturbance, Stream Incision, and Channel Evolution: the Roles Of Excess Transport Capacity and Boundary Materials in Controlling Channel Response. Geomorphology, v. 79, n. 3-4, p. 361-383, 2006.

SMITH, D. G. Fluvial geomorphology: where do we go from here? Geomorphology, v. 7, n. 1-3, p. 251-262, 1993.

SOTCHAVA, V. B. O estudo de geossistemas. Revista IG-USP, v. 16, p. 1-52, 1977.

STANFORD J. A; WARD J. V. An Ecosystem Perspective of Alluvial Rivers: Connectivity and the Hyporheic Corridor. Journal of the North American Benthololgical Society, v. 12, p. 48-60, 1993.

STRAHLER, A. N. Hypsometric (Area-Altitude) - Analysis of Erosional Topography. Geological Society of America Bulletin, v. 63, n. 10, p. 1117-1142, 1952.

VANNOTE, R. L.; MINSHALL, G. W.; CUMMINS, K. W.; SEDELL, J. R.; CUSHING, C. E. The River Continuum Concept. Canadian Journal of Fish and Aquatatic Science, v. 37, p. 130-137, 1985.

VAUDOR, L. Trends in Publications in Fluvial Geomorphology Over Two Decades: a Truly New Era in the Discipline Owing to Recent Technological Revolution? Geomorphology, v. 248, p. 489-500, 2015.

VITTE, A. C. A Construção da Geomorfologia no Brasil. Revista Brasileira de Geomorfologia, v. 12, n. 3, p. 91-108, 2011.

VESTENA, L. R.; KOBIYAMA, M. A geometria fractal da rede de drenagem da bacia hidrográfica do Caeté, Alfredo Wagner-SC. Revista Árvore, v. 34, n. 4, p. 661-668, 2010.

WARD, J. V. The four-dimensional nature of lotic ecosystems. Journal of North American, Benthological Society, v. 8, p. 2-8, 1989.

; STANFORD, J. A. The Serial Discontinuity Concept: Extending the Model to floodplains Rivers. Regulated Rivers: Research \& Management, v. 10, p. 159-168, 1995.

WHITEHEAD, M. Environmental Transformation. A Geography of Anthropocene. Londres: Routledge, 2014.

WILLIAMS, P. B. River Engineering Versus River Restoration. ASCE Wetlands. A Keynote Speech Given at Engineering and River Restoration Conference. Reno, Nevada, 2001. 
WOHL, E. Time and the Rivers Flowing: Fluvial Geomorphology Since 1960. Geomorphology, v. 216, p. 263-282, 2014.

WOLMAN, M. G.; LEOPOLD, L. B. River Flood Plains: Some Observations on Their Formation. U.S. Geological Survey Professional Paper 282C. U.S. Government Printing Office, Washington, DC, p. 87-109, 1957.

; GERSON, R. Relative Scales of Time and Effectiveness of Climate in Watershed Geomorphology. Earth Surface Processes and Landforms, v. 3, p. 189-208, 1978.

WU, J. G.; LOUCKS, O. L. From Balance of Nature to Hierarchical Patch Dynamics: a Paradigm Shift in Ecology. Quarterly Review of Biology, v. 70, n. 4, p. 439-466, 1995. 